\title{
Sources of the Ultra-High Energy Cosmic Rays (UHECRs) (Part II)
}

\author{
J. Wirsich \\ Reichsstrasse 107, Berlin, Germany \\ Email: astrometrie@gmx.de
}

How to cite this paper: Wirsich, J. (2017) Sources of the Ultra-High Energy Cosmic Rays (UHECRs) (Part II). Open Access Library Journal, 4: e3107.

http://dx.doi.org/10.4236/oalib.1103107

Received: November 28, 2016

Accepted: January 9, 2017

Published: January 12, 2017

Copyright $\odot 2017$ by author and Open Access Library Inc.

This work is licensed under the Creative Commons Attribution International License (CC BY 4.0).

http://creativecommons.org/licenses/by/4.0/

\begin{abstract}
It has recently been postulated that protons are accelerated up to energies of $E_{p} \geq 10^{19} \mathrm{eV}$ from nearby Seyfert galaxies which harbor Kerr holes in the center of their hosts. Here, we point out that the relativistic force equation of protons is governed by the Lorentz force when using the post-Newtonian approximation. The synchroton emission from nonthermal relativistic protons has to be ruled out. We carefully discuss the origin of the magnetic field close to the event horizon of a black hole.
\end{abstract}

\section{Subject Areas}

Modern Physics

\section{Keywords}

Cosmic Electrodynamics, Cosmic Rays, Black Hole Physics, Galaxies Active: Individual: NGC 4051

\section{Introduction}

The origin of the ultra-high energy cosmic rays (UHECRs) with energies greater than $10^{19} \mathrm{eV}$ is an important topic in astrophysics. The sources of the UHECRs have to be very powerful, because they have to be able to accelerate cosmic rays to very high energies. The sources would have to be located in the range of distances of $d<100 \mathrm{Mpc}$ : cosmic rays from more distant sources would have to lose energy when interacting with cosmic microwave background photons. This is the GZK cutoff (see e.g. [1]). The extragalactic protons not deflected by magnetic fields would have to point to their sources.

In Wirsich [1], we have presented the new idea that some local Seyfert galaxies that host supermassive Kerr holes in their nucleus are candidates of astrophysical sources of the UHECRs. In extension of the paper [1], we present in this 
study the relativistic force equation of a proton (Section 2) by applying the post-Newtonian approximation. In Section 3, we discuss the origin of the magnetic field, by emphasizing the dynamical character of black holes (spin-paradigm). In Section 4, we discuss our results. Finally, Section 5 presents our conclusions.

\section{The Post-Newtonian Approximation (PNA)}

The full relativistic equation of a motion of a particle of mass $m_{p}$, charge $e_{0}$, velocity $\boldsymbol{v}$ and momentum $\boldsymbol{p}$ including the Lorentz force is [2]

$$
\frac{\mathrm{d} \boldsymbol{p}}{\mathrm{d} t}=e_{0}(\boldsymbol{E}+\boldsymbol{v} \times \boldsymbol{B})+m_{p} \gamma_{p} \boldsymbol{g}+\underline{\underline{H} \boldsymbol{p}}+\boldsymbol{f}_{\mathrm{rad}}
$$

where $\boldsymbol{p}=m_{p} \gamma_{p} \boldsymbol{v}$ is the paricle momentum, $\gamma_{p}$ the Lorentz factor, $\boldsymbol{g}$ the gravitational acceleration and $\underline{\underline{H}}$ is the tensor of the gravimagnetic force [3]. Force $\boldsymbol{f}_{\text {rad }}$ is the radiation reaction force of ultra-relativistic particles in an external electromagnetic field given by

$$
\boldsymbol{f}_{\text {rad }}=\frac{2 e_{0}^{4} \gamma_{p}^{2}}{3 m_{p}^{2}}\left[(\boldsymbol{E}+\boldsymbol{v} \times \boldsymbol{B})^{2}-[\boldsymbol{v} \cdot(\boldsymbol{E}+\boldsymbol{v} \times \boldsymbol{B})]^{2}\right] \frac{\boldsymbol{v}}{|\boldsymbol{v}|} .
$$

$\boldsymbol{E}$ is the electric field and $\boldsymbol{B}$ the magnetic field. $\boldsymbol{f}_{\text {rad }}$ represents the energy loss by synchrotorn radiation and the curvature energy ("tidal radiation") [4]. In Figure 1 of Wirsich [1] we have chosen a scale length of $10 r_{g}$, where $r_{g}=\frac{G M}{c^{2}}$ is the gravitational radius and $M$ the mass of the black hole. $G$ means the gravitational constant. An approximation like PNA signifies that scale length 10 $r_{g}$ is large compared to $r_{+}$, but in the range of the capture radius of a solar-type star $r_{C}=2 r_{g}-11 r_{g}$ for a Kerr hole with a mass of $10^{8} \mathrm{M} \odot$ that is rotating with a spin-parameter near $a \approx 0.9$ [5]. On the other hand, the value of $10 r_{g}$ is well beyond the event horizon of

$$
r_{+}=\frac{G M}{c^{2}}+\left[\left(\frac{G M}{c^{2}}\right)^{2}-a^{2}\right]^{1 / 2}
$$

of a Kerr hole. $r=r_{+}$means that a particle entering it is unable to escape. Therefore, $r_{+}$is defined as the horizon of a Kerr hole, which forms a boundary around the black hole singularity. Penrose [6] has shown that naked singularities without a horizon cannot form.

The PNA means moreover that $10 r_{g}$ is well below the influence radius $r_{\text {inf }}=\frac{G M}{\sigma^{2}}$, where $\sigma$ is $t$ the velocity dispersion of stars in any direction [7] [8]. The calculation of the PNA-metric is given in [3] (see chapter 3 therein). We find far from the horizon $r_{+}$for the gravitational acceleration $\boldsymbol{g}$

$$
\boldsymbol{g} \approx-\frac{G M}{r^{2}} \boldsymbol{e}_{r}
$$

and for the field $\boldsymbol{H}$

$$
\boldsymbol{H} \approx-4 \frac{\boldsymbol{J}}{r^{3}}
$$


where $M$ is the mass of the black hole. Note that the angular momentum $\boldsymbol{J}$ is weak far from the black hole.

In conclusion, the first term in Equation (1) remains the leading form, since the second and the third term are small at $10 r_{g}$ compared to the Lorentz force, when using $10^{8} M_{\odot}$ for the mass of the black hole. On the other hand, neither a synchrotron radiation nor a curvature radiation of relativistic protons is ever observed, although several authors have postulated such an effect [9] [10] [11]. The $\mathrm{X}$ - and $\gamma$-ray non-thermal emission of blazars in the range of $10^{12} \mathrm{eV}$ are modelled as a sum of synchrotron and Compton radiation. In the hadronic models, the source of the synchrotron radiation has been related to relativistic protons. The calculated luminosities at $1 \mathrm{GeV}$ are in the range of $L_{\gamma} \approx 10^{45} \mathrm{erg} \times \mathrm{s}^{-1}$ only, while the observed luminosity is $L_{\gamma} \approx 10^{48} \mathrm{erg} \times \mathrm{s}^{-1} \quad$ [9]. Accordingly, hadronic models or proton blazar models have to be ruled out: roughly speaking, the radiation losses remain with the electrons, while the protons keep their high energy. This is plausible when studying Equation (2): factor $1 / m_{p}^{2}$ is to be substituated by $(1836)^{2} / m_{p}^{2}$ when using the Equation (2) for electrons ("leptonic models").The value 1836 is the ratio of $m_{p} / m_{e}$.

Furthermore, models for proton synchrotron radiation from hot disks around neutron stars are not able to produce the observed X-ray radiation of $L_{X} \approx 10^{36} \mathrm{erg} \times \mathrm{s}^{-1}$. On the other hand, nonthermal relativistic protons should be able to produce a luminosity of $L_{\text {syn. }} \approx 10^{33} \mathrm{erg} \times \mathrm{s}^{-1}$ in optical and UV bands [12]. However, nondetection of such effect signifies the lack of proton synchrotron radiaiton.

In all, the last term in Equation (1) is $f_{\text {rad }}=0$.

\section{The Origin of the Magnetic Field}

Studying Figure 1 in Wirsich [1], it appears that the magnetic field has its origin in the black hole. However, this is not the case, since this would contradict the non-hair theorem. On the other hand, in the Blandford-Znajek process, the black hole is interpreted as a membrane model which is equivalent to the curved spacetime paradigm [13]: in this model, the horizon of a black hole consists of a charged membrane. Here, the magnetic fields have their origin in the horizon of a black hole by accretion of the disk's plasma. Rougly speaking, the horizon of the rotating black hole has build up a magnetossphere (see [3], chapter 4 therein). Accordingly, a Kerr hole is able to produce an enormous power that goes into the energy of protons.

\section{Discussion}

It appears that jets are only driven from central engines and massive black holes in host galaxies of elliptical Hubble type. But there are some signatures that nuclear jets are common features in Seyfert galaxies [14]. The Seyfert galaxies from [1] show small radio jets only: e.g. NGC 4051 shows a radio jet scale length of 0.3 pc [15]. Furthermore, several Seyfert galaxies show a radio luminosity which has its origin in circumstellar starbursts ("superwinds driven by starbursts") [14]. 
The no-hair theorem tells us that a black hole is uniquely fixed by its mass, spin and charge even the black hole spins fast and is strongly deformed by its spin e.g. to Kerr radius $r_{+}$. On the other hand, black holes are viewed as $\underline{d y-}$ namic objects which are able to storing $29 \%$ of the rotational energy, magnetic extraction of the rotational energy (horizon as a conducting membrane), emit gravitatonal waves and allow an escape of thermal quanta ("evaporation of black holes"). All of these processes take place in the horizon and explain the name event horizon, which covers the singularity. The event horizon guarantees the virginity of the singularity.

\section{Conclusions}

The strongly increasing loss of radiation energy at high redshifts signifies that protons with their ultrahigh energies have their origin at a depth of a few Mpc. Therefore, cosmic ray protons of $E_{p} \geq 1.5 \times 10^{19} \mathrm{eV}$ have their source in nearby Seyfert galaxies that harbor Kerr holes with a spin-parameter of $a \geq 0.9$ [1] ("charged-particle-astronomy").

The relativistic force equation of the protons is governed by the Lorentz force (see Equation (10) in [1]). The rotation energy of the Kerr hole is completely converted into the energy of protons. The radiation losses via synchrotron radiation and curvature radiation are $f_{\text {rad }}=0$.

We have discussed the origin of the magnetic field in connection with the membrane-paradigm [13]. Confirmation of the postulation that spinning black holes eject relativistic protons is a challenge to observation; evidence could be provided, e.g., from nearby Seyfert galaxies that contain Kerr holes in the center of their hosts.

\section{Acknowledgements}

The author is very grateful to Dr. Wolfgang Hasse (Technischen Universität Berlin and Wilhelm-Foerster-Sternwarte Berlin) and to Kevin Klinik (Technische Universität Berlin and Wilhelm-Foerster-Sternwarte Berlin) for their help in converting my manuscript into LaTeX version.

\section{References}

[1] Wirsich, J. (2015) Sources of the Ultra-High Energy Cosmic Rays (UHECRs) (Part I). Open Journal of Modern Physics, 2.

[2] Neronov, A. and Aharonian, F.A. (2007) Production of TeV Gamma Radiation in the Vicinity of the Supermassive Black Hole in the Giant Radio Galaxy M87. Astrophysical Journal, 671, 85-96. https://doi.org/10.1086/522199

[3] Thorne, K.S., Price, R.H. and Douglas, D.A. (1986) Black Holes: The Menbrane Paradigm. Yale University Press, New Haven, 73.

[4] Landau, L.D. and Lifschiz, E.M. (1963) Lehrbuch der Theoretischen Physik. Volume 2, Akademie-Verlag, Berlin, 189.

[5] Merritt, D. (2012) Dynamics and Evolution of Galactic Nuclei. Princeton University Press, Princeton and Oxford, 74. 
[6] Penrose, R. (1969) Nuovo Cimento, 1, 252-276.

[7] Gualandris, A. and Merritt, D. (2012) Long-Term Evolution of Massive Black Hole Binaries. IV. Mergers of Galaxies with Collisionally Relaxed Nuclei. Astrophysical Journal, 744, 74-94. https://doi.org/10.1088/0004-637X/744/1/74

[8] Merritt, D. (2012) Dynamics and Evolution of Galactic Nuclei. Princeton University Press, Princeton and Oxford, 19.

[9] Palma, N.I., Boettcher, M., de la Calle, I., et al. (2011) Multiwavelength Observations of the Gamma-Ray Blazar Pks 0528+134 in Quiescence. Astrophysical Journal, 735, 60-77. https://doi.org/10.1088/0004-637X/735/1/60

[10] Sikora, M., et al. (2009) Constraining Emission Models of Luminous Blazar Sources. Astrophysical Journal, 704, 38-50. https://doi.org/10.1088/0004-637X/704/1/38

[11] Zacharopoulou, O., et al. (2013) Modeling the Hard TeV Spectra of Blazars 1ES 0229+200 and 3C 66A with an Internal Absorption Scenario. Astrophysical Journal, 738, 157-165. https://doi.org/10.1088/0004-637X/738/2/157

[12] Yang, J. and Yi, I. (1999) Signatures of Energetic Protons in Hot Accretion Flows: Synchrotron Coolingof Protons in Strongly Magnetized Pulsars. Astrophysical Journal, 516, L21-L24. https://doi.org/10.1086/311985

[13] Straumann, N. (1988) The Membrane Model of Black Holes and Applications in Black Holes: Theory and Observations. Springer, New York, 111.

[14] Baum, S.A., O’Dea, C.P., Dallacassa, D., de Bruyn, A.G. and Pedlar, A. (1993) Kiloparsec-Scale Radio Emission in Seyfert Galaxies: Evidence for Starburst-Driven Superwinds? Astrophysical Journal, 419, 553. https://doi.org/10.1086/173508

[15] Maitra, D., Miller, J.M., Markoff, S. and King, A. (2011) A Jet Model for the Broadband Spectrum of the Seyfert 1 Galaxy NGC 4051. Astrophysical Journal, 735, 107. https://doi.org/10.1088/0004-637X/735/2/107

[16] Kerr, R.P. (1963) Gravitational Field of a Spinning Mass as an Example of Algebraically Special Metrics. Physical Review Letters, 11, 237-238. https://doi.org/10.1103/PhysRevLett.11.237

[17] Shapiro, S.D. and Teukolsky, S.A. (1983) Black Holes, White Dwarfs and Neutron Stars. John Wiley and Sons, New York. https://doi.org/10.1002/9783527617661

[18] Hawking, S.W. (1976) Black Holes and Thermodynamics. Physical Review D, 13, 191-197. https://doi.org/10.1103/PhysRevD.13.191

[19] Tchekhvskoy, A., Narayan, R. and McKinney, J.C. (2010) Black Hole Spin and the Radio Loud/Quiet Dichotomy of Active Galactic Nuclei. Astrophysical Journal, 711, 50-63. https://doi.org/10.1088/0004-637X/711/1/50

[20] Miller, J.M., Miller, M.C. and Reynolds, S. (2011) The Angular Momenta of Neutron Stars and Black Holes as a Window on Supernovae. Astrophysical Journal, 731, L5. https://doi.org/10.1088/2041-8205/731/1/L5

[21] Ulmer, A. (1994) Super-Eddington Radiation Transfer in Soft Gamma Repeaters. Astrophysical Journal, 437, L111-L114. https://doi.org/10.1086/187695 


\section{Appendix}

\section{The Kerr hole and the membrane paradigm}

A New Zealand mathematician, named Roy Kerr, discovered a solution to Einstein's field equation [16].

The gravitational field is described by metric tensor $g_{\mu \nu}$. It is used to give the invariant interval $d s^{2}$ of a spacetime instead of the metric tensor.

The Kerr solution is a metric that describes the time independent gravitational field of collapsed objects. The Kerr metric takes the form given in equation (12.7.1) of [17]. As the Schwarzschild metric, the Kerr metric has a singularity at $r=r_{+}$(see Equation (3)). When $a \rightarrow 0$, the Kerr metric reduces to the Schwarzschild metric. $r=r_{-}$is called the Cauchy horizon.

The nature of the Kerr geodesics plays an important role in the process e.g. of extraction of energy from black holes. An example for this is given by the Blandford-Znajek process (BL-process) (see ref. [18] in [1]). The mechanism supposes that a rotating black hole accretes material from a magnetised disk. Here, the event horizon is viewed as a spinning conductor. As the disk accretes gas into the hole, it carries magnetic field lines with it. The gas attached the horizon, but do not enter it. In this way the conductor ("membrane") of matter holds magnetic field through electric currents and charges: a magnetosphere is set up in the vicinity of the black hole.

As explained by the BZ-process, energy entering near the horizon, a strong flux of angular momentum leaves the horizon. That ensures that energy is extracted from the black hole. The membrane viewpoint e.g. motivate Hawking [18] to construct a connection between laws of thermodynamics and black hole mechanics (rotating or not). A fine review of the membrane model is given in [19] of [1].

In 1986, the model of the BZ-process was recalculated by Straumann (see ref. [20] in [1]). He confirmed the values of the magnetic and electric fields given in Equation (11) of ref. [1]. Numerical simulations show that the BZ-process can be very efficient, depending on the spin of the black hole [19]. In addition to the observations of local Seyfert galaxies (see Table 1 in [1]), several X-ray binaries harbor black holes with a high Kerr parameter a (see e.g. Table 1 in [20]).

Let us calculate the magnetic field at a distance of $10 r_{g}$ from the center of the black hole. For this, we use the scattering process of free electrons in an ionized plasma ("Thomson scattering").

The luminosity in which the radiation pressure on free electrons balances gravity is the well known Eddington limit [17]

$$
L_{E}=\frac{4 \pi G M m_{p} c}{\sigma_{T}},
$$

where $M$ is the mass of the black hole, $m_{p}$ the mass of the proton and $\sigma_{T}=6.65 \times 10^{-25} \mathrm{~cm}^{2}$ means the Thomson cross section. For particle density $n_{E} \quad\left(\right.$ in $\mathrm{cm}^{-3}$ ) we have at $10 r_{g}$

$$
n_{E}=\frac{1}{\sigma_{T} 10 r_{g}}=10^{10} .
$$


This is the density at $10 r_{g}$, where $r_{g}=\frac{G M}{c^{2}}$ and $M=10^{8} M_{\odot}$. Assumig a balance between the energy densities

$$
\frac{B^{2}}{8 \pi}=n_{E} m_{p} c^{2},
$$

we find at $10 r_{g}$ the result

$$
B \approx 1.95 \times 10^{4} \mathrm{G} .
$$

However, it cannot be excluded that scattering process is dominated by cyclotron scattering instead of Thomson scattering. Unfortunately, the opacity of cyclotron scattering near a magnetosphere of a Kerr hole is unknown. Instead, we use data from magnetospheres of neutron stars. We find at $10 r_{g}$ the result

$$
B \approx 6.19 \times 10^{5} \mathrm{G},
$$

where $\sigma_{c y l}=0.00 \sigma_{T}$ was used [21].

In summary, our approximations lead to the results in the range of those of Straumann (see Equation (11) in [1]) who has solved the relativistic Maxwell equations.

Submit or recommend next manuscript to OALib Journal and we will provide best service for you:

- Publication frequency: Monthly

- 9 subject areas of science, technology and medicine

- Fair and rigorous peer-review system

- Fast publication process

- Article promotion in various social networking sites (LinkedIn, Facebook, Twitter, etc.)

- Maximum dissemination of your research work

Submit Your Paper Online: Click Here to Submit

Or Contact service@oalib.com 